\title{
Ramsey numbers and the size of graphs
}

\author{
Benny Sudakov*
}

\begin{abstract}
For two graph $H$ and $G$, the Ramsey number $r(H, G)$ is the smallest positive integer $n$ such that every red-blue edge coloring of the complete graph $K_{n}$ on $n$ vertices contains either a red copy of $H$ or a blue copy of $G$. Motivated by questions of Erdős and Harary, in this note we study how the Ramsey number $r\left(K_{s}, G\right)$ depends on the size of the graph $G$. For $s \geq 3$, we prove that for every $G$ with $m$ edges, $r\left(K_{s}, G\right) \geq c(m / \log m)^{\frac{s+1}{s+3}}$ for some positive constant $c$ depending only on $s$. This lower bound improves an earlier result of Erdős, Faudree, Rousseau, and Schelp, and is tight up to a polylogarithmic factor when $s=3$. We also study the maximum value of $r\left(K_{s}, G\right)$ as a function of $m$.
\end{abstract}

\section{Introduction}

For two graphs $H$ and $G$, the Ramsey number $r(H, G)$ is the smallest positive integer $n$ such that any red-blue coloring of the edges of the complete graph $K_{n}$ on $n$ vertices contains either a red copy of $G$ or a blue copy of $H$. If $H=G$ we usually denote $r(G, G)$ by $r(G)$. The problem of determining or accurately estimating Ramsey numbers is one of the central problems in modern Combinatorics, and it has received a considerable attention, see, e.g., [10], 5]. In most cases the Ramsey number is estimated in terms of the order (number of vertices) of the the graph. However, in the early 80's Erdös and Harary asked about the relation between $r(H, G)$ and the sizes (number of edges) of the graphs $H$ and $G$.

The first partial answers to this general problem were obtained by Erdős, Faudree, Rousseau, and Schelp [8]. They determined up to a constant factor the minimum value of $r(G)$ for all graphs of size $m$ and showed that the order of magnitude of this minimum is $\Theta(m / \log m)$. They also proved that for fixed $s \geq 3$ there exist constants $c_{1}, c_{2}$ such that

$$
c_{1} m^{\frac{s}{s+2}}<\min _{e(G)=m} r\left(K_{s}, G\right)<c_{2} m^{\frac{s-1}{s}} .
$$

\footnotetext{
*Department of Mathematics, Princeton University, Princeton, NJ 08544, and Institute for Advanced Study, Princeton. E-mail: bsudakov@math.princeton.edu. Research supported in part by NSF CAREER award DMS-0546523, NSF grant DMS-0355497, USA-Israeli BSF grant, Alfred P. Sloan fellowship, and the State of New Jersey.
} 
This estimate is not sharp and there is a large gap between the upper and lower bounds even when the complete graph is a triangle $(s=3)$. In this case Erdős [6] conjectured that the upper bound $O\left(\mathrm{~m}^{2 / 3}\right)$ is closer to the truth. Our first result improves the bounds from [8] and confirms this conjecture.

Theorem 1.1 Let $s \geq 3$ and let $G$ be a graph with $m$ edges. Then there exists a constant $c$ depending only on such that

$$
r\left(K_{s}, G\right) \geq c(m / \log m)^{\frac{s+1}{s+3}} .
$$

On the other hand, there exists a graph $G$ of size $m$ such that $r\left(K_{s}, G\right) \leq O\left(m^{\frac{s-1}{s}} / \log ^{\frac{s-2}{s}} m\right)$.

In particular, when $s=3$ this determines the minimum value of $r\left(K_{3}, G\right)$ for all graphs $G$ of size $m$ up to a polylogarithmic factor and shows that

$$
\Omega\left(\frac{m^{2 / 3}}{\log ^{2 / 3} m}\right)<\min _{e(G)=m} r\left(K_{3}, G\right)<O\left(\frac{m^{2 / 3}}{\log ^{1 / 3} m}\right) .
$$

Another specific question which is part of the general problem mentioned in the first paragraph is to bound the maximum value of $r(H, G)$ when the graphs $H$ and $G$ have a given size. One of the basic results in Ramsey Theory is the fact that for the complete graph $G$ with $m$ edges, $r(G)=2^{\Theta(\sqrt{m})}$. A conjecture of Erdös [7] (see also [5]) asserts that there is an absolute constant $c$ such that for any graph $G$ with $m$ edges, $r(G) \leq 2^{c \sqrt{m}}$. This conjecture is still open. For bipartite graphs it was proved by Alon, Krivelevich and Sudakov [3]. They also show that for general graphs $G$ with $m$ edges, $r(G) \leq 2^{c \sqrt{m}} \log m$ for some absolute positive constant $c$. For the first off-diagonal case Harary conjectured and Sidorenko [15] proved that $r\left(K_{3}, G\right) \leq 2 m+1$ for any graph $G$ of size $m$ without isolated vertices. This inequality is best possible, since $r\left(K_{3}, G\right)=2 m+1$ for any tree with $m$ edges. Thus for $s=3$ the graphs which maximize $r\left(K_{3}, G\right)$ are very sparse. However, for $s>3$ Erdös conjectured that exactly the opposite is true and to maximize $r\left(K_{s}, G\right)$ over all graphs with $m$ edges one should make $G$ as nearly complete as possible. Motivated by this question we obtain the following general upper bound on $r\left(K_{s}, G\right)$ for graphs $G$ of size $m$.

Theorem 1.2 Let $s \geq 3$ and let $G$ be a graph with $m$ edges and without isolated vertices. Then there exists a constant $c$ depending only on s such that

$$
r\left(K_{s}, G\right) \leq c m^{\frac{s-1}{2}} / \log \frac{s-3}{2} m .
$$

When $G$ is a clique with $m$ edges it is known by the result of Ajtai, Komlós and Szemerédi [1] that $r\left(K_{s}, G\right) \leq O\left(m^{\frac{s-1}{2}} / \log ^{s-2} m\right)$. Hence our estimate has, up to a polylogarithmic factor, similar order of magnitude to the best known upper bound for off-diagonal Ramsey numbers of cliques.

The rest of this short note is organized as follows. In the next section we present proofs of our main results. The final section contains some concluding remarks and open problems. Throughout the paper we make no attempts to optimize various absolute constants. To simplify the presentation, we often omit floor and ceiling signs whenever these are not crucial. All logarithms are in the natural base $e$. 


\section{Proofs}

To prove Theorem 1.1 we use an approach developed by Krivelevich [13, which is based on probabilistic arguments together with large deviation inequalities. The fist inequality we need is a standard bound of Chernoff (see Appendix A in 2] ) which states that if $X$ is a binomially distributed random variable with parameters $m$ and $p$, then for every $a>0$

$$
\mathbb{P}[X-p m<-a] \leq e^{-\frac{a^{2}}{2 p m}} .
$$

Another large deviation bound, which we use in the proof, was obtained by Erdös and Tetali [9] (see also Chapter 8.4 in [2]).

Let $\Omega$ be a finite set (in our instance it is the set of edges of a complete graph) and let $R$ be a random subset of $\Omega$ such that $\mathbb{P}[\omega \in R]=p_{\omega}$ independently for all $\omega \in \Omega$. Let $C_{i}, i \in I$ be subsets of $\Omega$, where $I$ is some finite index set. For every $C_{i}$ we define $A_{i}$ to be the event that $C_{i} \subseteq R$. Let $X_{i}$ be the indicator random variable of event $A_{i}$ and let $X=\sum_{i \in I} X_{i}$ be the number of $C_{i} \subseteq R$. Finally, let $X_{0}$ be the maximum number of pairwise disjoint subsets $C_{i}$ which belong to $R$. Obviously, $X_{0} \leq X$. Let $\mu$ be the expectation of $X$; then Erdős and Tetali gave the following bound on the possible size of $X_{0}$ :

$$
\mathbb{P}\left[X_{0} \geq k\right] \leq \frac{\mu^{k}}{k !} \leq\left(\frac{e \mu}{k}\right)^{k}
$$

Proof of Theorem 1.1, Let $n=\frac{1}{3 s^{3}}(\mathrm{~m} / \log m)^{\frac{s+1}{s+3}}$ and consider coloring the edges of the complete graph $K_{n}$ such that each edge is colored randomly and independently red with probability $p=\frac{1}{3 s} n^{-\frac{2}{s+1}}$ and blue with probability $1-p$. Let $G_{1}, \ldots, G_{t}$ be all subgraph of $K_{n}$ which are isomorphic to $G$. The number of such subgraphs $t$ is clearly bounded by the number of injective functions from $V(G)$ to $K_{n}$, which in turn is at most the number of permutations on $n$ elements. Thus $t \leq n$ !. For every subgraph $G_{i}$, let $X_{i}$ be the random variable that counts the number of red edges in $G_{i}$. By definition, $X_{i}$ is binomially distributed with parameters $m$ and $p$. Hence, by Chernoff's inequality

$$
\mathbb{P}\left[X_{i}<m p / 2\right]=\mathbb{P}\left[X_{i}-m p<-m p / 2\right] \leq e^{-m p / 8}
$$

Also for every subgraph $G_{i}$ define $Y_{i}$ to be the number of red cliques of order $s$ which share at least one edge with $G_{i}$. Since $G_{i}$ has $m$ edges, the number of $s$-cliques sharing at least one edge with

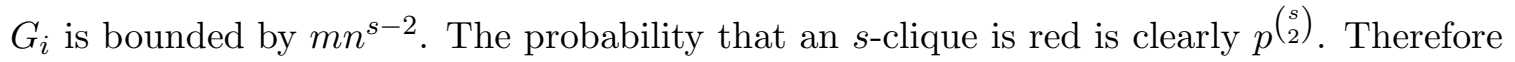

$$
\mathbb{E}\left[Y_{i}\right] \leq m n^{s-2} p^{\left(\begin{array}{l}
s \\
2
\end{array}\right)}=m p n^{s-2} p^{\frac{(s+1)(s-2)}{2}}=\left(\frac{1}{3 s}\right)^{\frac{(s+1)(s-2)}{2}} m p \leq \frac{m p}{9 s^{2}} .
$$

Let $Y_{i}^{\prime}$ be the maximum number of edge disjoint red $s$-cliques which share at least one edge with $G_{i}$ Then by the Erdös-Tetali inequality we have that

$$
\mathbb{P}\left[Y_{i}^{\prime} \geq m p / s^{2}\right] \leq\left(\frac{e \mathbb{E}\left[Y_{i}\right]}{m p / s^{2}}\right)^{m p / s^{2}} \leq\left(\frac{e}{9}\right)^{m p / s^{2}} \leq e^{-m p / s^{2}} .
$$


By definition of $n$ and $p$ we have that $m p=\left(3 s^{3} n\right)^{\frac{s+3}{s+1}} p \log m \geq s^{2} n \log n>8 n \log n$. Therefore the probability that for some index $i$ either $X_{i}<m p / 2$ or $Y_{i}^{\prime} \geq m p / s^{2}$ is bounded by $t\left(e^{-m p / 8}+e^{-m p / s^{2}}\right) \leq$ $2 n ! n^{-n}=o(1)$. In particular there exists a red-blue edge coloring of $K_{n}$ such that for every $1 \leq i \leq t$, subgraph $G_{i}$ contains at least $m p / 2$ red edges and there are at most $m p / s^{2}$ edge disjoint red $s$-cliques each sharing at least one edge with $G_{i}$.

Fix such a coloring and let $\Gamma$ be the subgraph of red edges in it. Also let $\mathcal{C}$ be the maximum (under inclusion) collection of edge disjoint cliques of order $s$ in $\Gamma$. Recolor edges in all cliques from $\mathcal{C}$ by blue and denote the remaining red graph by $\Gamma^{\prime}$. Note that by recoloring we removed from $\Gamma$ the maximum collection of edge disjoint $s$-cliques. Thus $\Gamma^{\prime}$ contains no clique of order $s$. On the other hand in every subgraph $G_{i}$ we changed the color of at most $\left(\begin{array}{l}s \\ 2\end{array}\right) m p / s^{2}<m p / 2$ red edges. Since originally $G_{i}$ had at least $m p / 2$ red edges, we obtain that every subgraph of $K_{n}$ isomorphic to $G$ still has at least one red edge. This implies that new coloring contains no blue copy of $G$ and no red copy of $K_{s}$ and completes the proof of the first statement of the theorem.

To prove the second part, let $G$ be the union of $\frac{2 m}{k(k-1)}$ vertex disjoint cliques of order $k=$ $m^{1 / s}(\log m)^{\frac{s-2}{s}}$. By definition, the number of edges in $G$ is at least $m$. To estimate the Ramsey number $r\left(K_{s}, G\right)$ we use the result of Ajtai, Komlós and Szemerédi [1] (see also Theorem 12.17 in [4]) which bounds off-diagonal Ramsey numbers. They prove that there exists a constant $c$ such that $r\left(K_{s}, K_{k}\right) \leq c \frac{k^{s-1}}{\log ^{s-2} k}$. Let

$$
n=c \frac{k^{s-1}}{\log ^{s-2} k}+2 m /(k-1)=O\left(m^{\frac{s-1}{s}} / \log \frac{s-2}{s} m\right)
$$

and consider any red-blue edge coloring of the complete graph $K_{n}$. We can assume that there is no red $s$-clique, or else we are done. Then, since $n \geq r\left(K_{s}, K_{k}\right)$, we can find a blue clique of order $k$. Delete it from the graph and continue this process. Note that as long as we deleted less than $\frac{2 m}{k(k-1)}$ cliques of order $k$ the remaining number of vertices is still larger than $r\left(K_{s}, K_{k}\right)$ and we can find a new blue $k$-clique. In the end we will find at least $\frac{2 m}{k(k-1)}$ blue cliques of size $k$, i.e., a copy of $G$. This implies that $r\left(K_{s}, G\right) \leq O\left(m^{\frac{s-1}{s}} / \log \frac{s-2}{s} m\right)$ and completes the proof.

Proof of Theorem 1.2, We prove the theorem by induction on $s$. Consider the case $s=3$. Although one can use results from [8] and [15] to show that $r\left(K_{3}, G\right) \leq O(m)$ we include here the simple proof that $r\left(K_{3}, G\right) \leq 3 m$ for the sake of completeness. Clearly we can assume that $G$ is connected, since $r\left(K_{3}, G_{1} \cup G_{2}\right) \leq r\left(K_{3}, G_{1}\right)+r\left(K_{3}, G_{2}\right)$. Hence the number of vertices of $G$ is at most $m+1$. Let $n=3 m$ and suppose that the edges of $K_{n}$ are red-blue colored with no red triangle. Pick the vertex with maximum red degree in this coloring and let $X,|X|=t$, be the set of its red neighbors. Note that all the edges inside $X$ are blue, since there is no red triangle. Partition the vertices of $G$ into two sets $V(G)=V^{\prime} \cup V^{\prime \prime}$, where $V^{\prime}$ consists of the $t$ vertices with the highest degree. Since the sum of the degrees in $G$ is $2 m$, we have that all the vertices in $V^{\prime \prime}$ have degree at most $2 m /(t+1)$. Now we will find the blue copy of $G$ as follows. Embed the vertices of $V^{\prime}$ into $X$ arbitrarily, and then embed the vertices of $V^{\prime \prime}$ one by one. Given a vertex $v \in V^{\prime \prime}$, let $Y$ be the set 
of vertices of $K_{n}$ where we already embedded neighbors of $v$. Since the maximum red degree in the coloring is $t$ and $|Y| \leq d(v) \leq 2 m /(t+1)$ we have that $K_{n}$ contains at least $3 m-t|Y| \geq m+1-|Y|$ vertices which are adjacent to all vertices in $Y$ by blue edges. As the total number of vertices of $G$ is at most $m+1$, one such vertex is still unoccupied and can be used to embed $v$. Continuing this process we find a blue copy of $G$.

Now suppose $s>3$ and by induction we have that $r\left(K_{s-1}, G\right) \leq c_{1} m^{\frac{s-2}{2}} / \log ^{\frac{s-4}{2}} m$. Let $n=$ $c_{2} m^{\frac{s-1}{2}} / \log \frac{s-3}{2} m$, where $c_{2}$ is a sufficiently large constant which depends on $c_{1}$ and which we fix later. Consider a red-blue coloring of the complete graph $K_{n}$ such that there is no red copy of $K_{s}$. If there is a vertex which has at least $d=c_{1} m^{\frac{s-2}{2}} / \log \frac{s-4}{2} m$ red neighbors, then this set cannot contain a red copy of $K_{s-1}$. Therefore by the induction hypothesis it will contain a blue copy of $G$ and we are done. Thus we can assume that the maximum degree in the red subgraph of $K_{n}$ is at most $d$. Set $k=\sqrt{m \log m}$. It is easy to check that, by definition, $n=\Omega\left(\frac{k^{s-1}}{\log ^{s-2} k}\right)$. Therefore, by choosing $c_{2}$ large enough and using the result of Ajtai, Komlós and Szemerédi [1] on Ramsey numbers, we get that $n \geq r\left(K_{s}, K_{k}\right)$. Hence there exists a set $X$ of $k$ vertices which spans only blue edges. Again partition the vertices of $G$ into two sets $V(G)=V^{\prime} \cup V^{\prime \prime}$, where $V^{\prime}$ consists of the $k$ vertices with the highest degree. Since the sum of the degrees in $G$ is $2 m$, we have that all the vertices in $V^{\prime \prime}$ have degree at most $2 m /(k+1)$. Embed the vertices of $V^{\prime}$ into $X$ arbitrarily, and then embed the vertices of $V^{\prime \prime}$ one by one as follows. Given a vertex $v \in V^{\prime \prime}$, let $Y$ be the set of vertices of $K_{n}$ where we already embedded neighbors of $v$. Since the maximum red degree in the coloring is $d$ and $|Y| \leq d(v) \leq 2 m /(k+1)$, by choosing sufficiently large $c_{2}$, we have that there are at least

$$
n-d|Y| \geq n-2 m d /(k+1)>c_{2} m^{\frac{s-1}{2}} / \log \frac{s-3}{2} m-\frac{2 m}{\sqrt{m \log m}}\left(c_{1} m^{\frac{s-2}{2}} / \log \frac{s-4}{2} m\right)>2 m
$$

vertices in $K_{n}$ which are adjacent to all vertices in $Y$ by blue edges. Note that the total number of vertices of $G$ is at most $2 m$, as it has no isolated vertices. Therefore there exists an unoccupied vertex of $K_{n}$ which is connected to all vertices in $Y$ by blue edges. This vertex can be used to embed $v$. In the end of this procedure we obtain a blue copy of $G$. This completes the proof of the theorem.

\section{Concluding remarks}

- Let $H$ be a graph with $v_{H} \geq 3$ vertices and $e_{H}$ edges. The density $\rho(H)$ of $H$ is defined as $\rho(H)=\frac{e_{H}-1}{v_{H}-2}$. Define also

$$
\rho^{*}(H)=\max _{H^{\prime} \subseteq H} \rho\left(H^{\prime}\right) .
$$

For example, for the complete graph of order $s$ we have $\rho^{*}\left(K_{s}\right)=\frac{s+1}{2}$. The arguments in the proof of Theorem 1.1 can be used to obtain the following more general result. Since the proof of this statement does not require new ideas and contains somewhat tedious computations we omit it here. 
Theorem 3.1 Let $H$ be a fixed graph. Then there exists a constant $c$ depending only on $H$ such that for every graph $G$ with $m$ edges,

$$
r(H, G) \geq c(m / \log m)^{\frac{\rho^{*}}{1+\rho^{*}}} .
$$

In addition to the triangle, this result is nearly tight when $H$ is the complete bipartite graph $K_{p, q}$ with $q \gg p$. Indeed it is easy to check from the definition that if $p$ is fixed and $q \rightarrow \infty$ then $\rho^{*}\left(K_{p, q}\right) \rightarrow p$. Therefore for every $p$ and $\epsilon>0$ there exists $q$ such that $\frac{\rho^{*}\left(K_{p, q}\right)}{1+\rho^{*}\left(K_{p, q}\right)}>\frac{p}{1+p}-\epsilon$. Thus, from Theorem 3.1 we have that $r\left(K_{p, q}, G\right) \geq \Omega\left(m^{\frac{p}{1+p}-\epsilon}\right)$ for every $G$ with $m$ edges. On the other hand, from the result of Kövari, Sós, Turán [12] that $K_{p, q}$-free graphs on $n$ vertices can have at most $O\left(n^{2-1 / p}\right)$ edges, it follows that such a graph has an independent set of size $\Omega\left(n^{1 / p}\right)$. This implies that $r\left(K_{p, q}, K_{k}\right) \leq O\left(k^{p}\right)$ (see also [3, 14] for slightly better estimate). Using this bound together with the argument from the proof of the second part of Theorem 1.1. we can show that if $G$ is the disjoint union of $\Theta\left(m^{\frac{p-1}{p+1}}\right)$ cliques of order $m^{1 /(p+1)}$ then $r\left(K_{p, q}, G\right) \leq O\left(m^{\frac{p}{1+p}}\right)$.

- For $s=3$ the lower bound in Theorem 1.1 is tight up to a multiplicative factor of $\log ^{1 / 3} \mathrm{~m}$. It would be very interesting to close this gap. We think that our upper bound in (11) is closer to the truth and there exists an absolute constant $c$ such that $r\left(K_{3}, G\right) \geq \mathrm{cm}^{2 / 3} / \log ^{1 / 3} m$ for every graph $G$ of size $m$. To prove this one might try to use an approach based on the semi-random method which was developed by Kim [11] to determine the asymptotic behavior of Ramsey numbers $r\left(K_{3}, K_{k}\right)$.

- It would be interesting to extend an upper bound in Theorem 1.2 to the general case when $H$ and $G$ are arbitrary graphs with sizes $t$ and $m$ and with no isolated vertices. We conjecture that if $t$ is fixed and $m$ is sufficiently large then

$$
r(H, G) \leq m^{O(\sqrt{t})} .
$$

This estimate if true is tight up to a constant in the exponent, since the known lower bounds (see [16, 13]) on off-diagonal Ramsey numbers imply that $r(H, G) \geq m^{\Omega(\sqrt{t})}$ when $H$ and $G$ are complete graphs with $t$ and $m$ edges respectively. In [3] it was proved that if $H$ is a graph with chromatic number $\ell$ and maximum degree $d \geq \ell$ then for all sufficiently large $m$, $r\left(H, K_{2 m}\right) \leq m^{\ell d}$. Using this estimate it is easy to obtain the following partial result, which shows that our conjecture holds if the chromatic number of $H$ is a fixed constant.

Proposition 3.2 Let $H$ and $G$ be two graphs with no isolated vertices such that the size of $G$ is $m$, the size of $H$ is $t$ and $H$ has chromatic number $\ell \geq 2$. Then there exists a constant $c$ depending only on $\ell$ such that for sufficiently large $m, r(H, G) \leq m^{c \sqrt{t}}$.

Sketch of proof. We use induction on $t$. Let $v$ be the vertex of maximum degree in $H$. Since $G$ has $m$ edges, it has at most $2 m$ vertices. Therefore, if the maximum degree of $H$ is at most 
$2 \sqrt{t}$, it follows from the above cited estimate in 3 , that $r(H, G) \leq m^{2 \sqrt{t} \ell}$. Otherwise, the degree of $v$ in $H$ is larger than $2 \sqrt{t}$. Delete it and denote $H_{1}=H \backslash\{v\}$. This graph has $t_{1} \leq t-2 \sqrt{t}$ edges and $\sqrt{t_{1}} \leq \sqrt{t}-1$.

Let $n=m^{2 \sqrt{t} \ell}$ and consider red-blue coloring of the edges of the complete graph $K_{n}$. Since $G$ has at most $2 m$ vertices, we can assume that there is no red $K_{2 m}$ in this coloring. Therefore, by Turán's theorem, there is a vertex $x$ in $K_{n}$, whose blue degree is at least $n / 2 m \gg m^{(2 \sqrt{t}-2) \ell} \geq$ $m^{2 \sqrt{t_{1}} \ell}$. Let $U$ be the set of blue neighbors of $x$. Clearly, this set contains no blue copy of $H_{1}$. Now we can use induction to conclude that it contains a red copy of $G$.

\section{References}

[1] M. Ajtai, J. Komlós and E. Szemerédi, A note on Ramsey numbers, J. Combinatorial Theory Ser. A 29 (1980), 354-360.

[2] N. Alon and J. Spencer, The Probabilistic Method, 2nd ed., Wiley, New York, 2000.

[3] N. Alon, M. Krivelevich and B. Sudakov, Turán numbers of bipartite graphs and related Ramseytype questions, Combinatorics, Probability and Computing 12 (2003), 477-494.

[4] B. Bollobás, Random Graphs, 2nd ed., Cambridge Studies in Advanced Mathematics, 73, Cambridge University Press, Cambridge, 2001.

[5] F. Chung and R. Graham, Erdős on graphs. His legacy of unsolved problems, A K Peters Ltd., Wellesley, MA, 1998.

[6] P. Erdős, Solved and unsolved problems in combinatorics and combinatorial number theory, Proc. 12th Southeastern Conference on Combinatorics, Graph Theory and Computing, Vol. I, Congr. Numer. 32 (1981), 49-62.

[7] P. Erdős, On some problems in graph theory, combinatorial analysis and combinatorial number theory, in: Graph theory and combinatorics (Cambridge, 1983), Academic Press, London, 1984, $1-17$.

[8] P. Erdős, R. Faudree, C. Rousseau and R. Schelp, A Ramsey problem of Harary on graphs with prescribed size, Discrete Math. 67 (1987), 227-233.

[9] P. Erdős and P. Tetali, Representation of integers as the sum of $k$ terms, Random Structures and Algorithms 1 (1990), 245-261.

[10] R. Graham, B. Rothschild and J. Spencer, Ramsey theory, $2^{\text {nd }}$ ed., Wiley, New York, 1990.

[11] J.H. Kim, The Ramsey number $R(3, t)$ has order of magnitude $t^{2} / \log t$, Random Structures Algorithms 7 (1995), 173-207. 
[12] T. Kövari, V.T. Sós and P. Turán, On a problem of K. Zarankiewicz, Colloquium Math. 3 (1954), 50-57.

[13] M. Krivelevich, Bounding Ramsey numbers through large deviation inequalities, Random Structures and Algorithms 7 (1995), 145-155.

[14] Y. Li and W. Zang, Ramsey numbers involving large dense graphs and bipartite Turán numbers, J. Combinatorial Theory Ser. B 87 (2003), 280-288.

[15] A. Sidorenko, The Ramsey number of an $n$-edge graph versus triangle is at most $2 n+1, J$. Combinatorial Theory Ser. B 58 (1993), 185-196.

[16] J. Spencer, Asymptotic lower bounds for Ramsey numbers, Discrete Mathematics 20 (1977), 69-76. 